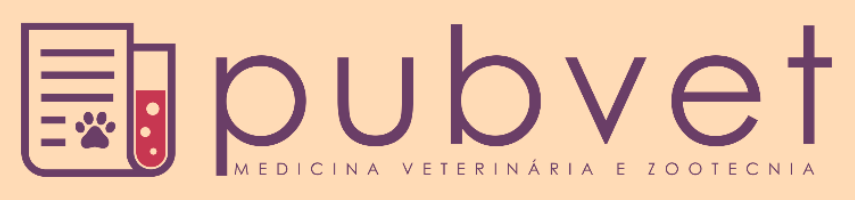

ISSN 1982-1263

https://doi.org/10.31533/pubvet.v13n3a298.1-4

\title{
Síndrome do granuloma lepróide em um cão na cidade de Pelotas: Relato de caso
}

\author{
Francisco de Assis Araújo Camelo Júnior ${ }^{1 *} \bullet$, Caroline Castagnara Alves $^{2} \bullet$, Maria \\ Gabriela de Mello Fonseca ${ }^{\bullet}{ }^{\bullet}$, Mateus de Azevedo Soares $^{2}{ }^{\bullet}$, Maurício Andrade \\ Bilhalva $^{2}{ }^{\circ}$, Risciela Salardi Alves de Brito ${ }^{30}$
}

${ }^{I}$ Médicos Veterinários, autônomos, Pelotas/RS-Brasil;

${ }^{2}$ Acadêmicos de Medicina Veterinária, Universidade Federal de Pelotas, Pelotas/RS- Brasil;

${ }^{3}$ Mestranda do Departamento de Clínicas Veterinárias, Universidade Federal de Pelotas, Pelotas/RS-Brasil.

*Autor para correspondência, E-mail: junior_camelo01@hotmail.com

Resumo. A Síndrome do granuloma lepróide canino é uma enfermidade incomum de etiologia micobacteriana, provavelmente subdiagnosticada. Clinicamente, as lesões cutâneas ocorrem preferencialmente em regiões mais susceptíveis a picadas de insetos, como orelhas e cabeça. Na maioria dos casos relatados, os cães acometidos apresentam bom estado geral de saúde e é encontrado como um achado clínico, sem envolvimento de outros órgãos. O diagnóstico pode ser bastante sugestivo pelo padrão racial. Porém, a confirmação do diagnóstico deve ser realizada através de biópsia ou histopatologia. $\mathrm{Na}$ maioria dos casos a citologia aspirativa por agulha fina é o exame de escolha, pela praticidade, rapidez e segurança. A maioria dos casos são auto-limitantes e regride espontaneamente entre um e três meses após o aparecimento inicial, mas geralmente cursam com infecção cutânea crônica, localizada ou generalizada. Dessa forma, o objetivo do presente trabalho é relatar o caso de um cão com diagnóstico definitivo de síndrome do granuloma lepróide canina na cidade de Pelotas, esclarecendo as condutas feitas com o paciente desde o atendimento até o regresso da lesão localizada.

Palavras chave: citológico, clínica, histopatológico, lepra, tratamento

\section{Leproid granuloma syndrome in a dog in the city of Pelotas: Case report}

Abstract. The leproid canine granuloma syndrome is an uncommon disease of mycobacterial etiology, probably underdiagnosed. Clinically, cutaneous lesions occur preferentially in regions more susceptible to insect bites, such as ears and head. In most of the reported cases, the affected dogs present good general health and are found as a clinical finding, without involvement of other organs. The diagnosis can be quite suggestive by the racial pattern. However, confirmation of the diagnosis should be made through biopsy or histopathology. In most cases the CAAF is the exam of choice, for practicality, speed and safety. Most cases are self-limiting and regress spontaneously between one and three months after the initial onset, but usually occur with localized or generalized chronic skin infection. Thus, the objective of the present study is to report the case of a dog with definitive diagnosis of canine leprosy granuloma syndrome in Pelotas city, clarifying the behaviors made with the patient from the care to the return of the localized lesion.

Keywords: cytological, clinical, histopathological, leprosy, treatment 


\section{Síndrome del granuloma leproso en un perro en la ciudad de Pelotas: Relato de caso}

Resumen. El Síndrome del granuloma leproso canino es una enfermedad inusual de
etiología micobacteriana, probablemente subdiagnosticada. Clínicamente, las lesiones
cutáneas se presentan preferentemente en regiones más susceptibles a picaduras de
insectos, como orejas y cabeza. En la mayoría de los casos reportados, los perros
acometidos presentan buen estado general de salud y se encuentra como un hallazgo
clínico, sin implicación de otros órganos. El diagnóstico puede ser bastante sugestivo por
el patrón racial. Sin embargo, la confirmación del diagnóstico debe realizarse a través de
biopsia o histopatología. En la mayoría de los casos la citología aspirativa por aguja fina es
el examen de elección, por la practicidad, rapidez y seguridad. La mayoría de los casos son
autolimitantes y reduce espontáneamente entre uno y tres meses después de la aparición
inicial, pero generalmente cursan con infección cutánea crónica, localizada o generalizada.
De esta forma, el objetivo del presente trabajo es relatar el caso de un perro con diagnóstico
definitivo de síndrome del granuloma leproso canino en la ciudad de Pelotas, aclarando las
conductas hechas con el paciente desde la atención hasta el regreso de la lesión localizada

Palabras clave: citología, clínica, histopatológico, lepra, tratamiento

\section{Introdução}

A Síndrome do granuloma lepróide canino é uma enfermidade incomum de etiologia micobacteriana, provavelmente subdiagnosticada (Conceição et al., 2011; Foley et al., 2002; Malik et al., 1998). O agente etiológico é uma micobacteria sapróbia de baixa patogenicidade e virulência tendo predileção por áreas corporéas de temperatura baixa (Gross et al., 2008; Gross et al., 2009). Clinicamente, as lesões cutâneas ocorrem preferencialmente em regiões mais susceptíveis a picadas de insetos, como orelhas e cabeça (Malik et al., 1998). Essas alterações se iniciam por nódulos firmes, circunscritos, alopécicos e indolores, que envolvem a derme e o tecido subcutâneo, podendo ocorrer uni ou bilateralmente (Malik et al., 2001). $\mathrm{Na}$ maioria dos casos relatados, os cães acometidos apresentam bom estado geral de saúde e é encontrado como um achado clínico, sem envolvimento de outros órgãos. Além disso, as lesões causam irritação e desfiguração do tecido, podendo ser infectadas secundariamente por Staphylococcus intermedius (Greene et al., 1993). O diagnóstico pode ser bastante sugestivo pelo padrão racial, normalmente de raça definida, grande porte e mais comum na raça Boxer (Larsson, 1996), também pelas características e distribuição das lesões, que acometem principalmente regiões auriculares, cabeça e membros, podendo ser uni ou bilaterais (Foley et al., 2002). Porém, a confirmação do diagnóstico deve ser realizada através de biópsia ou histopatologia. No caso da realização da biópsia por citologia por agulha fina (CAAF), foi utilizada uma coloração de Ziehl Nielsen, onde serão visualizados bacilos álcool ácidos resistentes, em número variável, no interior dos macrófagos ou no centro dos granulomas (Foley et al., 2002). Enquanto que no histopatológico será visto um infiltrado de células inflamatórias difusas por macrófagos, plasmócitos, neutrófilos e as vezes linfócitos próximos as glândulas anexas. Já com a coloração de panótico os bacilos ficam descorados no interior de macrófagos (Charles et al., 1999; Santoro et al., 2008). Na maioria dos casos a CAAF é o exame de escolha, pela praticidade, rapidez e segurança. Mesmo reduzindo a acurácia (em comparação com o histopatológico), o exame citológico é mais recomendado devido a ser pouco invasivo (Malik et al., 1998; Charles et al., 1999). Há poucos relatos disponíveis de tratamentos para a Síndrome do granuloma lepróide canina (Malik et al., 2006). A maioria dos casos são autolimitantes e regridem espontaneamente entre um e três meses após o aparecimento inicial, mas geralmente cursam com infecção cutânea crônica, localizada ou generalizada. Assim, o tratamento adequado seria o uso da antibioticoterapia e o acompanhamento com um especialista em dermatologia para avaliação da lesão, regresso do nódulo e condição clínica do paciente (Malik et al., 2006). Dessa forma, o objetivo do presente trabalho é relatar o caso de um cão com diagnóstico definitivo de síndrome do granuloma lepróide canina em Pelotas, esclarecendo as condutas feitas com o paciente desde o atendimento até o regresso da lesão localizada.

\section{Relato de caso}


Foi atendido um cão macho, da raça Pitibull, com aproximadamente um ano e cinco meses, pesando $23 \mathrm{~kg}$, com histórico de nódulos na região dorsal de ambos os pavilhões auriculares de evolução progressiva. Todos os parâmetros clínicos gerais avaliados estavam sem alterações. No exame clínico dermatológico foram observados nódulos auriculares bilaterais com aproximadamente 1 a $5 \mathrm{~cm}$ de diâmetro (Figura 1), consistência firme, alopécicos, não aderidos a cartilagem e com aumento progressivo relatado pelos tutores.
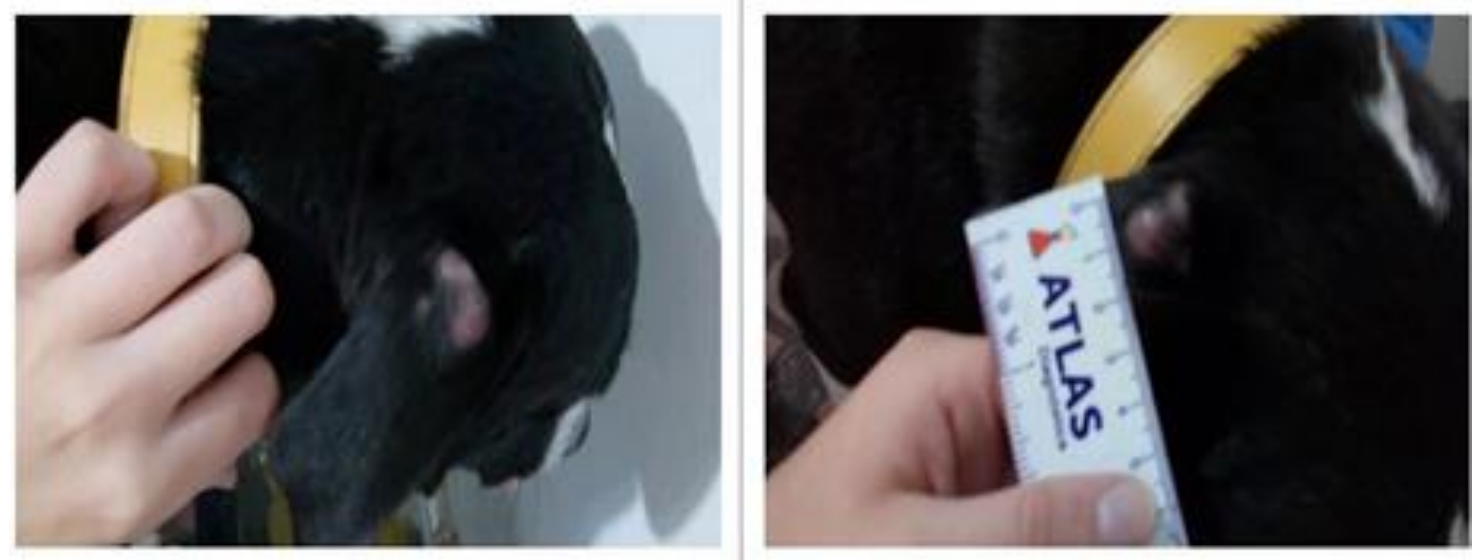

Figura 1. Nódulos auriculares bilaterais com aproximadamente um cm de diâmetro.

Foi realizada a citologia aspirativa por agulha fina (CAAF) como método complementar de diagnóstico. Após, foi encaminhado para o Laboratório Veterinário de Análises Clínicas, em Pelotas. Pela citologia foi detectada baixa celularidade, composta por neutrófilos e macrófagos e ocasional presença de plasmócitos e fibroblastos. Além disso, foram encontrados vários bacilos corados negativamente (compatíveis com Mycobacterium spp.) dentro do citoplasma de macrófagos e também extracelularmente. Assim, o exame foi sugestivo de inflamação mista, associada à infecção por Mycobacterium spp, bactéria responsável pela enfermidade. Após o resultado do exame, foi estipulado um tratamento com antibioticoterapia da classe das fluorquinolonas, enrofloxacina via oral de 250 miligramas, um comprimido, duas vezes ao dia durante 21 dias. Foi indicado retorno após 15 dias para reavaliação.

\section{Resultados e discussão}

Após 15 dias de tratamento, o paciente retornou até a clínica para uma reavaliação e pode-se notar que o aumento de volume visto inicialmente nos pavilhões auriculares havia diminuído. Além de ressaltar que o estado geral do mesmo encontrava-se hígido, dessa forma, podendo-se afirmar que o tratamento proposto foi eficiente. Corroborando com a literatura, onde Farias et al. (2008) afirma que o uso das fluorquinolonas é eficiente para essa afecção. Outros tratamentos indicados pela literatura são as associações medicamentosas, como com claritromicina $(7,5$ a $12,5 \mathrm{mg} / \mathrm{kg})$, doxiciclina $(5$ a 7,5 $\mathrm{mg} / \mathrm{kg}$ ) e rifampicina (10 a $15 \mathrm{mg} / \mathrm{kg}$ ), todos com tratamento de um a dois meses, sendo mais barato o uso de doxiciclina com rifampicina. Existem outros métodos de tratamento para a cura da micobactéria, como é o caso da ressecção cirúrgica. É uma forma eficiente, embora podendo ocorrer cura espontânea em seis meses. O prognóstico é excelente mesmo sem o tratamento terapêutico (Foley et al., 2002), mas o uso dos antibióticos pode ser uma boa alternativapara profilaxia de infecções cutâneas que em alguns estágios dessa doença pode ocorrer. Cabe ressaltar que a ressecção cirúrgica confirmará também o diagnóstico da doença, pois a amostrar extraída pode ser encaminhado para histopatológico.

Dentre os diagnósticos diferenciais, podem-se citar lesões nodulares neoplásicos ou não, como tricoblastoma, histiocitoma cutâneo canino, plasmocitoma cutâneo, mastocitoma, bem como granuloma estéril idiopático (Hargis \& Ginn, 2009) e as micoses subcutâneas. Assim, as avaliações citológicas e histopatológicas das lesões geralmente estabelecem o diagnóstico definitivo, evitando condutas terapêuticas erradas. Cabe ressaltar que mesmo essa enfermidade tenha uma casuística alta para raças definidas, como Boxers, de médio e grande porte e de animais com pelagem mais curta, não se pode descartar os demais. Por isso, é de grande importância ter o conhecimento dos sinais clínicos apresentado pelos pacientes, a associação com as diversas enfermidades que cursam com essas alterações, a 
atualização dos exames complementares, a aplicação do tratamento ideal e o acompanhamento período com o veterinário responsável.

\section{Conclusão}

A síndrome do granuloma lepróide é uma doença incomum na clínica médica de pequenos animais. As lesões apresentadas podem ser comuns a outras enfermidades sendo necessária a confirmação diagnóstica por método complementar. O tratamento com Enrofloxacina de $250 \mathrm{mg}$, um comprimido, duas vezes ao dia e por 21 dias foi eficiente na redução dos sinais clínicos dermatológicos.

\section{Referências bibliográficas}

Charles J., Martin P., Wigney D.I., Malik R. \& Love D.N. 1999. Cytology and histopathology of canine leproid granuloma syndrome. Australian. Veterinary. Journal. 77(12):799-803.

Conceição, L. G., Acha, L. M. R., Borges, A. S., Assis, F. G., Loures, F. H. \& Silva, F. F. (2011). Epidemiology, clinical signs, histopathology and molecular characterization of canine leproid granuloma: a retrospective study of cases from Brazil. Veterinary Dermatology, 22(3):249-256.

Farias, M. R., Casagrande, T. A. C., Werner, J. \& Cavalcante, C. Z. (2008). Retrospective study (19982007) of canine leproid granuloma syndrome in Curitiba, Brazil. in: World Congress of Veterinary Dermatology, Hong Kong. Proceeding. Hong Kong: the Asian Society of Veterinary Dermatology, p. 64.

Foley, J. E., Borjesson, D., 1 Gross, T., Rand, C., Needham, M. \& Poland, A. (2002). Clinical, microscopic, and molecular aspects of canine leproid granuloma in the United States. Veterinary Pathology, 39(2):234-239.

Greene, C. E., Samperio, J. O. \& Gómez, J. P. (1993). Enfermedades infecciosas: Perros y gatos. São Paulo: Editora Interamericana.

Gross, T. L., Ihrke, P. J., Walder, E. J. \& Affolter, V. K. (2008). Skin diseases of the dog and cat: clinical and histopathologic diagnosis. Oxford: John Wiley \& Sons.

Gross, T. L., Ihrke, P. J., Walder, J. E. \& Affolter, K. V. (2009). Doenças de pele do cão e do gato: diagnóstico clínico e histopatológico. São Paulo: Roca.

Hargis, A.M. \& Ginn, P.E. (2009). O Tegumento. In: Mcgavin, M.D.; Zachary, J. F. Bases da Patologia em Veterinária. Brasil: Elsevier. p.1252-1253.

Larsson, C. E. (1996). Dermatopatias alérgicas-hipersensibilidade medicamentosa (HM). Clínica Veterinária, 5(1):351-355.

Malik, R., Hughes, M. S., Martin, P. \& Wigney, D. (2006). Canine leproid granuloma syndrome (Canine leprosy). In C. E. Greene (Ed.), Infectious diseases of the dog and cat (Vol. 3, pp. 480-482). Saint Louis, USA: Saunders Elsevier.

Malik, R., Love, D. N., Wigney, D. I. \& Martin, P. (1998). Mycobacterial nodular granulomas affecting the subcutis and skin of dogs (canine leproid granuloma syndrome). Australian Veterinary Journal, 76(6):403-407.

Malik, R., Martin, P., Wigney, D., Swan, D., Sattler, P. S., Cibilic, D., . . . Hughes, M. S. (2001). Treatment of canine leproid granuloma syndrome: preliminary findings in seven dogs. Australian Veterinary Journal, 79(1):30-36.

Santoro, D., Prisco, M. \& Ciaramella, P. (2008) Cutaneous sterile granulomas/pyogranulomas, leishmaniasis anda mycobacterial infections. Journal of Small Animal Practice 49(1): 552-561

Recebido: 21 de fevereiro, 2019

Aprovado: 15 de março, 2019.

Publicado: 27 de março, 2019.

Licenciamento: Este artigo é publicado na modalidade Acesso Aberto sob a licença Creative Commons Atribuição 4.0 (CCBY 4.0), a qual permite uso irrestrito, distribuição, reprodução em qualquer meio, desde que o autor e a fonte sejam devidamente creditados. 